University of Nebraska - Lincoln

DigitalCommons@University of Nebraska - Lincoln

Papers in Biotechnology

Chemical and Biomolecular Engineering

Research and Publications

August 2005

\title{
Crosslinked chitosan: Its physical properties and the effects of matrix stiffness on chondrocyte cell morphology and proliferation
}

Anuradha Subramanian

Department of chemical Engineering,University of Nebraska Lincoln., asubramanian2@unl.edu

Hsin-YI Lin

Department of Chemical and Biomolecular Engineering, University of Nebraska-Lincoln

Follow this and additional works at: https://digitalcommons.unl.edu/chemeng_biotechnology

Part of the Biochemical and Biomolecular Engineering Commons

Subramanian, Anuradha and Lin, Hsin-YI, "Crosslinked chitosan: Its physical properties and the effects of matrix stiffness on chondrocyte cell morphology and proliferation" (2005). Papers in Biotechnology. 34. https://digitalcommons.unl.edu/chemeng_biotechnology/34

This Article is brought to you for free and open access by the Chemical and Biomolecular Engineering Research and Publications at DigitalCommons@University of Nebraska - Lincoln. It has been accepted for inclusion in Papers in Biotechnology by an authorized administrator of DigitalCommons@University of Nebraska - Lincoln. 


\section{Crosslinked chitosan: Its physical properties and the effects of matrix stiffness on chondrocyte cell morphology and proliferation}

\section{Anuradha Subramanian, Hsin-Yi Lin}

Abstract: Chitosan $[<(1-4)-2$ amino-2-deoxy-d-glucose], the natural polyaminosaccharide derived from $\mathrm{N}$-deacetylation of chitin $[<(1-4)-2$ acetamide-2-deoxy-d-glucose], has been shown to possess attractive biological and cell interactive properties. Recently chitosan and chitosan analogs have also been shown to support the growth and continued function of chondrocytes. In the present study, chitosan substrates are crosslinked with a functional diepoxide (1,4 butanediol diglycidyl ether) to alter its mechanical property, and the viability and proliferation of the canine articular chondrocytes seeded on the crosslinked surface are further assayed. Of interest is the impact of substrate stiffness on the growth and proliferation of articular canine chondrocytes. Cross linked scaffolds were also subjected to degradation by chitosanase to examine the impact of cross linking on enzymeassisted degradation. The hydrophilicity and compression modulus of the crosslinked surfaces were measured via contact-angle measurements and compression tests, respectively. Scanning electron microscopy (SEM) and fluorescent staining were used to observe the proliferation and morphology of chondrocyte cells on noncrosslinked and crosslinked surfaces. The crosslinked chitosan was found to be nontoxic to chondrocytes and more hydrophilic. Its compression modulus and stiffness increased, which may improve the scaffold resistance to wear and in vivo shrinkage once implanted. The increased stiffness also seemed to serve as an additional mechanical stimulus to promote chondrocyte growth and proliferation. The cell morphology on crosslinked scaffolds seen by SEM and fluorescent stain was the typical chondrocytic rounded shape. The method 
proposed provides a nontoxic way to increase the mechanical strength of the chitosan scaffolds.

Key words: cartilage tissue engineering; chitosan scaffold; chondrocytes; crosslinking; biomaterial mechanical testing

\section{INTRODUCTION}

The articular cartilage is the dense white tissue covering the articulating surfaces of bones. The regenerative capability of the articular cartilage is very limited when injured or damaged with aging. One approach to repair defected cartilage is to generate functional tissue by seeding functional chondrocyte cells in a biocompatible scaffold and then implant the cell-material complex to repair chondral defects. 1 The use of polymeric scaffolds to generate tissue-engineered cartilage has been extensively studied.2-8 Given the importance of GAG in stimulating chondrogenesis, use of GAG or GAG analogs as components of a cartilage tissue scaffold appears to be a logical approach. Chitosan has been reported to share some structural features with glycosaminoglycan, which occurs naturally in articular cartilage, and has been reported to support chondrogenesis and biosynthesis of markers associated with chondrocyte metabolism.7-10 By using a combination of freeze drying and leaching techniques, chitin/chitosan and its derivatives (carboxymethyl chitin and bicarboxymethyl chitosan) have been blended with hydroxyapatite to yield composites for use in bone-tissue engineering. 11 Chitosan sponges were made by freeze-drying tripolyphosphate crosslinked chitosan and loaded with platelet-derived growth factor. These scaffolds were evaluated for their ability to regenerate periondontal 
bone. Freeze-drying techniques have been used to prepare porous membranes and 3D scaffolds of chitosan for use in tissue engineering applications.12 In a previous study, nonporous chitosan membranes were modified with chondroitin sulfate (CSA) and CSAchitosan membranes were evaluated for their ability to support chondrogenesis.7,11 In a separate study, a scaffold in the form of an interpenetrating polymeric network was made from a mixture of collagen and chitosan.13 The biocompatibility of the chitosan-based scaffolds have been evaluated in mice, 14 where porous chitosan scaffolds (unseeded) were implanted in mice, and animals were sacrificed after 1, 2, 4, 8, or 12 weeks. Macroscopic inspection of the implantation site revealed no pathological inflammatory responses, gram staining and limulus assays revealed no evidence of infection or endotoxin, and lymphocyte proliferation assays and antibody responses indicated a low incidence of chitosan-specific reactions.14 This study serves to demonstrate that chitosanbased scaffolds had a high degree of in vivo biocompatibility in the animal model studied. The regulation of chondrocyte function (e.g., biosynthesis of Type II collagen, PG synthesis and down regulation of nitrous oxide production) by growth factors (TGF<), cytokines, and biomechanical forces (dynamic compression) has been demonstrated. 15-21 An area that has not been studied as well, but is critical to the understanding of cartilage function and maintenance during normal and disease states, is the regulation of chondrocyte function by an intrinsic mechanical environment or substrate mechanics. For example, osteoarthritis is brought about by the degradation of the collagen fibrils in the ECM, leading to a cartilage matrix with decreased tensile modulus, which in turn perhaps leads to decreased biosynthetic activity of chondrocytes. Chondrocytes, when seeded on substrates, are known to exert contractile forces, 22,23 which usually results in the shrinkage of the substrate, referred to as the chondrocyte-mediated contraction (CMC). The changes in size and microstructure of the implants/ substrates due to cell contraction may 
reduce the pore size and further impede the migration of cells into the scaffolds and free exchange of the nutrients and the metabolites.24 The potential of articular chondrocytes to exert a contractile force upon scaffolds and change the size and pore structure of the scaffolds has been reported both in vivo and in vitro.22-25 Collagen-based matrices, when seeded with chondrocytes, were reported to undergo up to $35 \%$ shrinkage.22,23 Noncrosslinked collagen sponges, when implanted in full-thickness osteochondral defects in canine models, were observed to undergo cell-driven contraction of the matrix and thus prevent complete fitting of scaffolds in the chondral defects.25,26 Preventive methods against mechanical changes of polymer scaffolds in the articular cartilage implant site have been studied.27,28 Crosslinking treatments have been used to enhance the mechanical properties of the polymeric scaffolds.22,23,29 -31 The effects of four crosslinking methods on the compressive stiffness of collagen-glycosaminoglycan (CG) matrices and the interaction between adult canine articular chondrocytes and the stiffened matrix have been evaluated. 22 Dehydrothermally treated and ultra violet irradiated matrices were found to be the most compliant, and 1-ethyl-3-(3-dimethylaminopropyl) carbodiimide (EDAC) and glutaraldehyde (GTA) -treated matrices were the stiffest. Over the 4-week culture period, the GTA and EDAC matrices (the stiff ones) resulted in the highest rate of cell proliferation. RGD-derivatized alginate hydrogels were crosslinked with $\mathrm{Ca} 2<$ and $\mathrm{Ba} 2<$ ions and were reported to provide 10 -fold higher chondrocyte attachment when compared to uncrosslinked alginate hydrogels.31 More recently,29 porous collagen scaffolds were crosslinked with EDAC in the presence of lysine; however, no obvious difference in resistance to contraction was observed over matrices crosslinked with EDAC. Furthermore, changes in stiffness upon crosslinking and the impact of the same of the morphology of seeded fibroblasts were not reported. The aim in this study was to chemically crosslink the $\mathrm{ONH} 2$ groups on the chitosan backbone and to modify the stiffness of 
chitosan-based scaffolds and substrates. Furthermore, increased stiffness is expected as a result of crosslinking to impact chondrocyte behavior, and to test the hypothesis that the rigidity of the matrix can itself serve as a mechanical signal and direct seeded chondrocytes to possess higher biosynthetic activity of crosslinked matrices.

\section{MATERIALS AND METHODS}

\section{Reagents}

All chemicals were of analytical grade or better. Chitosan with a degree of deacetylation of 81.7 was purchased from Vansom (Redmond, WA) and was used without further purification. The diepoxide was purchased from Sigma Chemical Company (St. Louis, MO). Articular chondrocytes of canine origin (shoulder joints of dogs) were obtained from Dr. Mark Beatty, College of Dentistry, University of Nebraska Medical School (Lincoln, NE). Cellculture media and other reagents were obtained from Invitrogen. Mechanical tests were performed on an MTS Instrument available at the College of Dentistry (University of Nebraska Medical School, Lincoln, NE). SEMs were obtained on a JEOL-6100 microscope at the Center of Biotechnology (UNL, Lincoln, NE). All reagents were used without further purification. 


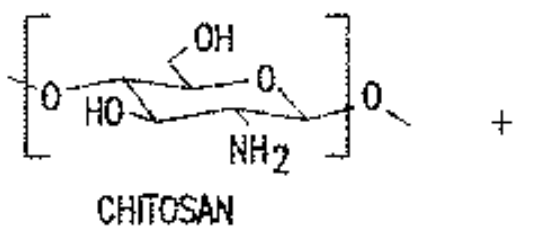

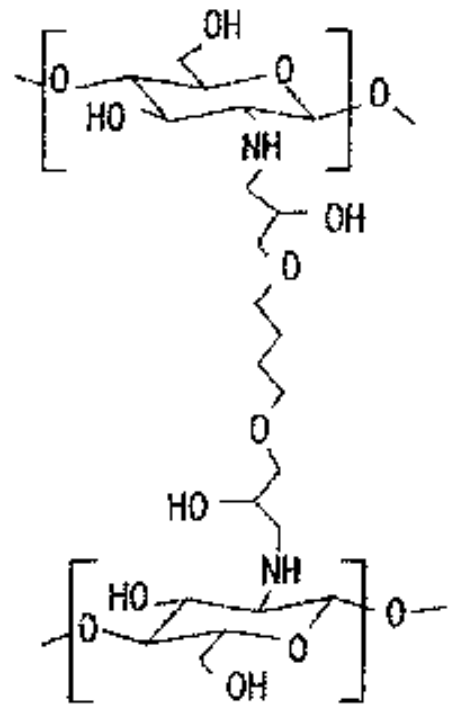

\section{Structure 1}

\section{Crosslinked chitosan units}

Figure 1. Schematic for the strategy for cross-linking with bifunctional epoxides. Chitosan-based films and scaffolds were first neutralized with $0.05 \mathrm{~N} \mathrm{NaOH}$, washed with sterile PBS, and dehydrated with isopropanol. The solid line indicates the chitosan backbone. Surfaces were incubated with a known stoichiometric excess of functional diepoxide. ${ }^{29}$ To obtain maximum crosslinking (Structure 1), the reaction was carried out at $50^{\circ} \mathrm{C}$ for $24 \mathrm{~h}$. The surfaces were washed in IP, PBS, and then used. The surfaces were washed in sterile PBS and stored until further use.

\section{Preparation of films and scaffolds}

A $2 \% \mathrm{w} / \mathrm{v}$ solution of chitosan $(81.7 \%$ deacetylated, MW $<276 \mathrm{kDa}$, Lot No. 01-CISQ1702, Vanson HaloSource, WA) in $1 \%$ acetic acid was freshly prepared prior to scaffold preparation. Glass slides (Fisher) were coated with 1-mL chitosan solution and air dried at room temperature. The chitosan coated slides were further treated in an $80^{\circ} \mathrm{C}$ oven over- 
night to prevent the detachment of the chitosan film. Dried films were then neutralized with $1 \mathrm{~N} \mathrm{NaOH}$ and sequentially rinsed with deionized water, air dried and stored until further use. For the preparation of scaffolds via freeze-dry and lyophilization (FDL), a 2-mL solution of $2 \%$ chitosan solution was prepared as mentioned earlier, and pipetted into each well of a 24-well tissue-culture polystyrene plate (TCP, Falcon). The samples were then frozen at $<20^{\circ} \mathrm{C}$ and the frozen samples were lyophilized for $24-36 \mathrm{~h}$. The scaffolds were then neutralized with $1 \mathrm{M} \mathrm{NaOH}$ and rinsed as detailed earlier. In order to preserve the shape (pores) of the scaffolds, the scaffolds were gradually dehydrated with a series of ethanol solutions (20-100\%) before air drying the scaffolds. The scaffold sample used in the cell seeding experiments was $0.8-1.0 \mathrm{~cm}$ in diameter and $0.3-0.5 \mathrm{~cm}$ in thickness.

\section{Crosslinking films and scaffold}

A diepoxide-based bifunctional linker32 (1,4 butanediol diglycidyl ether) was used to crosslink the chitosan chains via the reactive amino group on the chitosan backbone. The schematic of the crosslinking method is shown in Figure 1. By varying the molar stoichiometric excess of the diepoxide, films were prepared and scaffolds were freezedried with varying degrees of crosslinking. A crosslinking agent (1,4-butanediol diglycidyl ether, Sigma, MO) was added to isopropanol to make solutions in which the mole ratio of the diepoxide to the $\mathrm{NH} 2$ groups on the chitosan was $1 / 2$ to $1(0.5<), 1$ to $1(1<)$, and 5 to $1(5<)$. Controls $(0<)$ were samples submerged in plain isopropanol without adding the epoxide. Samples were placed in a $45^{\circ} \mathrm{C}$ shaker incubator at $60 \mathrm{rpm}$ for $16 \mathrm{~h}$. After $16 \mathrm{~h}$, samples were rinsed with isopropanol to remove excess ether and air dried. Prior to cell- 
seeding experiments, scaffolds and films were sequentially washed with sterile deionized water followed by sterile PBS.

\section{Cell culture}

All cells used in this study were passage 2 or 3 canine chondrocytes, harvested from the articular cartilage obtained from the shoulder joint of adult dogs. The cell-culture media used was RPMI $<10 \%$ fetal bovine serum supplemented with antibiotics and antimycotics (all cell culture reagents are from Invitrogen). Experiments with cells were maintained in a cell-culture incubator (Isotemp, Kendro, NC) at $37^{\circ} \mathrm{C}, 95 \%$ humidity and $5 \% \mathrm{CO} 2$.

\section{Surface hydrophilicity}

A contact angle system (model OCA $15<$, Dataphysics Co.) was used to test the hydrophilicity of the crosslinked films. Water was used as the liquid phase. Five microliters $(<L)$ of water was injected on the film surface at a rate of $3<\mathrm{L} / \mathrm{s}$. A Laplace-Young fitting was used to calculate the contact angle in degrees. For each epoxide to $(\mathrm{ONH} 2)$ molar ratio, three slides were used and six data points from each slide with error $<1.0$ were taken for average and standard-deviation calculation.

\section{Compression test}

Scaffolds were rehydrated in deionized water 1 day before the test. The test was performed on an Instron machine (model 1123, Instron, MA). The crosshead speed was 2 $\mathrm{mm} / \mathrm{min}$ and a 50-kgf load cell was used with Instron. Six specimens of each epox- 
ide/ONH2 mole ratio were tested at room temperature. The load (kgf) -displacement (millimeter) data were recorded by the computer software provided by Instron and converted to stress-strain curves to obtain elastic modulus $(\mathrm{kPa})$. No maximum compression strength was obtained because the hydrogel did not break when subject to compression. Films were tested according to the procedure detailed elsewhere.33

\section{Degradation with chitosanase}

The dry weight $(W 0)$ of the scaffolds was measured before the test. Chitosanase (EMD Biosciences) was dissolved in acetate solution (sodium acetate buffer: 200 mM, pH 4.5 plus $0.02 \%$ sodium azide) to a final concentration of $0.1 \mathrm{U} / \mathrm{mL}$. Scaffolds were submerged in chitosanase solution $(1: 100 \mathrm{w} / \mathrm{v})$ and incubated at $37^{\circ} \mathrm{C}$ with constant shaking over indicated periods of time $(1,2,4$, and $24 \mathrm{~h})$. Samples were removed from the enzyme solution after each time period and the scaffolds were rinsed with DI water. The samples were dried and weighed $(W f)$, and the percentage of degradation was calculated $\left[\left(W_{0}<\right.\right.$ $W f) / W 0<100 \%]$

Chondrocyte cell adhesion and viability test with MTT [3-(4,5-dimethylthiazol-2-yl)2,5- diphenyltetrazoliumbromide] assay

Glass slides coated with chitosan films were cut into $1<1-\mathrm{cm} 2$ squares and placed in the wells of 12-well TCP plates with the film facing upward. Samples were disinfected with 
$70 \%$ ethanol. Canine chondrocytes were then seeded on the films at $3<104 /$ film. Cells seeded on TCP served as controls. After 3 days, the cell-culture medium was removed and a $1 \mathrm{mg} / \mathrm{mL}$ solution of MTT was added to the cells. After $4 \mathrm{~h}$, the MTT solution was removed and isopropanol was added and held for $20 \mathrm{~min}$. The color of formazan salt was measured by an ELISA plate reader (ELx800, Biotek) at $595 \mathrm{~nm}$ (A595). Cell viability was calculated as a percentage of the TCP control [(Asample/Acontrol) $<100 \%]$.

\section{Cell morphology and proliferation (SEM and fluorescent stain)}

To observe the cells growing on two-dimensional substrate, chondrocyte cells were seeded at $1<104 / \mathrm{cm} 2$ on films that were not attached to slides (free-swelling) and cultured for 1 week before visualization by microscopy. At the end of the culture period, the medium was removed from each well, $300<\mathrm{L}$ of trypsin (Gibco) solution $(0.25 \%$ trypsin in sterile PBS) was added to each well, and the plates were held for $30 \mathrm{~min}$ for cells to detach. The solution in the well was pipetted up and down to get cells into suspension, and the cell counting was done with the use of a hemocytometer.33 Upon cell seeding, the seeded scaffolds were transferred to a fresh TCP plate, and prior to trypsinization the scaffolds were carefully removed to a new TCP. This step was done to avoid an increase in cell numbers arising from cells bound to the TCP plate. To quantify the total doublestranded DNA, the scaffolds were cut into small pieces, digested in $1 \mathrm{~mL}$ of a $6 \mathrm{rg} / \mathrm{mL}$ of papain solution for $6 \mathrm{~h}$ at $60^{\circ} \mathrm{C}$, and a PicoGreen ${ }^{\mathrm{TM}}$ DNA assay kit (Molecular Probes) was used according to manufacturer's specifications. To observe cells on a threedimensional matrix, scaffolds were placed in a 24-well TCP submerged in $2 \mathrm{~mL}$ of cell suspension with cell density of $1<105 / \mathrm{mL}$. After $24 \mathrm{~h}$, scaffolds were moved to another TCP with fresh media. Cells were cultured for a week before SEM and for 2 weeks before 
fluorescent stain. For SEM microscopy, cells were cross linked with $2.5 \%$ formaldehyde (Sigma) in phosphatebuffered saline (PBS), rinsed with deionized water, and gradually dehydrated with series of ethanol solutions. Hexamethyl disilazane (Fisher, PA) was used to remove $100 \%$ ethanol. Samples were sputter coated with Au-Pd before being examined under SEM (Hitachi, s3000N, Japan). For fluorescent staining, cells were fixed in $3.7 \%$ formaldehyde in PBS, penetrated with $0.1 \%$ Triton X-100 (Sigma, MO) in PBS, and stained with rhodamine-phalloidini (Molecular Probe) in $0.1 \%$ bovine serum albumin. $A$ confocal laser scanning microscope (BioRadi MRC1024ES, Hercules, CA) was used to examine the cells.

\section{Statistical analysis}

The numerical data from each experiment were the average from at least triplicate samples. The same experiments were repeated three times to ensure the repeatability of the methods used. A Student $t$ test was used for statistical analysis and the statistical differences were established as $p<0.05$.

\section{RESULTS AND DISCUSSION}

Although the impact of external stimuli (i.e., dynamic compression or hydrostatic pressure), and the presence of growth factors and cytokines on chondrocyte attachment, proliferation, and biosynthetic activity has been extensively studied, the impact of substrate stiffness on chondrocyte attachment and proliferation has only been studied recently.34 36 Chondrocyte-mediated contraction of matrices can be expected to impact the pore 
size and porosity, which, in turn, would impact the response to mechanical stimuli as well as chondrocyte biosynthetic and metabolic activity.24 In a previous study,22 crosslinking was shown to directly reduce the chondrocyte- mediated contraction of collagen-based matrices, wherein the stiffest matrix contracted by $30 \%$ and the complaint matrices contracted by $60 \%$. Interestingly, matrices with a higher-level contraction led to higher levels of Type II collagen biosynthesis when compared to stiffer matrices, possibly due to highdensity cultures in contracted matrices. Thus in this study the effects of crosslinking and matrix density on chondrocyte differentiation could not be delineated. Recently alginate matrices were first derivatized with RGD peptide and then cross- linked with $\mathrm{Ca} 2<$ and $\mathrm{Ba} 2<$ ions to increase the stiffness of the base alginate matrix.31 The rate and the extent of attachment were found to be dependent on the stiffness, and stiffer substrates were shown to impact chondrocyte morphology. However, in this study, the relative impact of the biochemical stimuli (RGD peptide) versus the intrinsic mechanical stimuli (i.e., matrix stiffness) could not be separated. Furthermore, the high levels of $\mathrm{Ca} 2<$ ions used to crosslink alginate may have modulated intracellular calcium levels, which has been reported to affect integrin expression and attachment in several cell types.37,38 Although $\mathrm{Ba} 2<$ has been reported to interfere with integrin function39 and block cell adhesion, 40 the use of $\mathrm{Ba} 2<$ to crosslink alginate surfaces has resulted in increased cell adhesion.31 In the present study, a change in the intrinsic mechanical environment was made by altering the morphological state of the matrix (planar film and scaffolds prepared via freeze drying and lyophilization), and the stiffness of the matrix was changed by crosslinking of the chitosan substrate. The intention here is to study the impact of intrinsic stimuli provided by morphologies or altered matrix stiffness independent of the stimuli provided by changes in matrix densities, or the presence of a bioactive peptide. It is known that the proposed reaction scheme (shown in Fig. 1) will result in both Structures 1 and 2, and that 
the ratio of the same can be controlled by an optimization of the reaction conditions, namely, the temperature and percent diepoxide in the reaction mixture. Briefly, the concentration of the terminal epoxide moiety (Structure 2) was estimated as function of reaction temperature and percent diepoxide, by sodium thiosulfate titration (data to be published). The present reaction optimization experiments have suggested that a high degree of crosslinking (Structure 1) can be obtained by carrying out the reactions at 45 to $60^{\circ} \mathrm{C}$, with less than $10 \%$ of free terminal epoxide groups (Structure 2). Infrared (FTIR) spectroscopy was used to determine the modification of the chitosan surface qualitatively. IR data were taken on desiccated chitosan films both before and after epoxide modification. The signature of the chitosan molecule can be significantly recognized from the intensity of the amine peak at wavelengths of $3300-3500 \mathrm{~cm}<1$ and the strong $\mathrm{NOH}$ stretch at 1560-1650 $\mathrm{cm}<1$, as seen from the IR spectrum shown in Figure 2(a). The IR spectrum of the diepoxide modified chitosan (1-molar excess of diepoxide) is shown in Figure 2(b). After the modification of chitosan with 1, 4 butanediol diglycidyl ether, the intensity of the characteristic amino peak at $3300-3500 \mathrm{~cm}<1$ was found to decrease, and a strong stretch at $1590 \mathrm{~cm}<1$ was observed, signifying the modification of the primary amine group on chitosan with the diepoxide. There is also an increase in the peak at $500 \mathrm{~cm}<1$ which is due to $\mathrm{COH} 2$ stretch. The peak $1060 \mathrm{~cm}<1$ is that from the $\mathrm{COOOH}$ resulting from the opening of the oxirane moiety by the primary amine group. These observations support the conclusion that chitosan was crosslinked at the terminal amino groups via the diepoxide linker. Similar FTIR spectra were obtained for films at other crosslinking ratios. Furthermore, it was observed that the crosslinked scaffolds/films were stable over the culture period (data not included). 


\section{Surface hydrophilicity (contact angle)}

The results, shown in Figure 3, showed that crosslinking the chitosan film with 1,4 butanediol diglycidylether decreased the contact angle and increased the hydrophilicity of the surface. Figure 3 shows a significant drop $(p<0.01)$ in contact angle from $0<$ to $0.5<$, but not between $0.5<$ and $1<$. The contact angle decreased again $(p<0.01)$ when the ether/amino ratio was raised to $5<$. Although the contact angles of crosslinked films were lower than the noncrosslinked films, the hydrophilicity of the crosslinked films studies were similar.
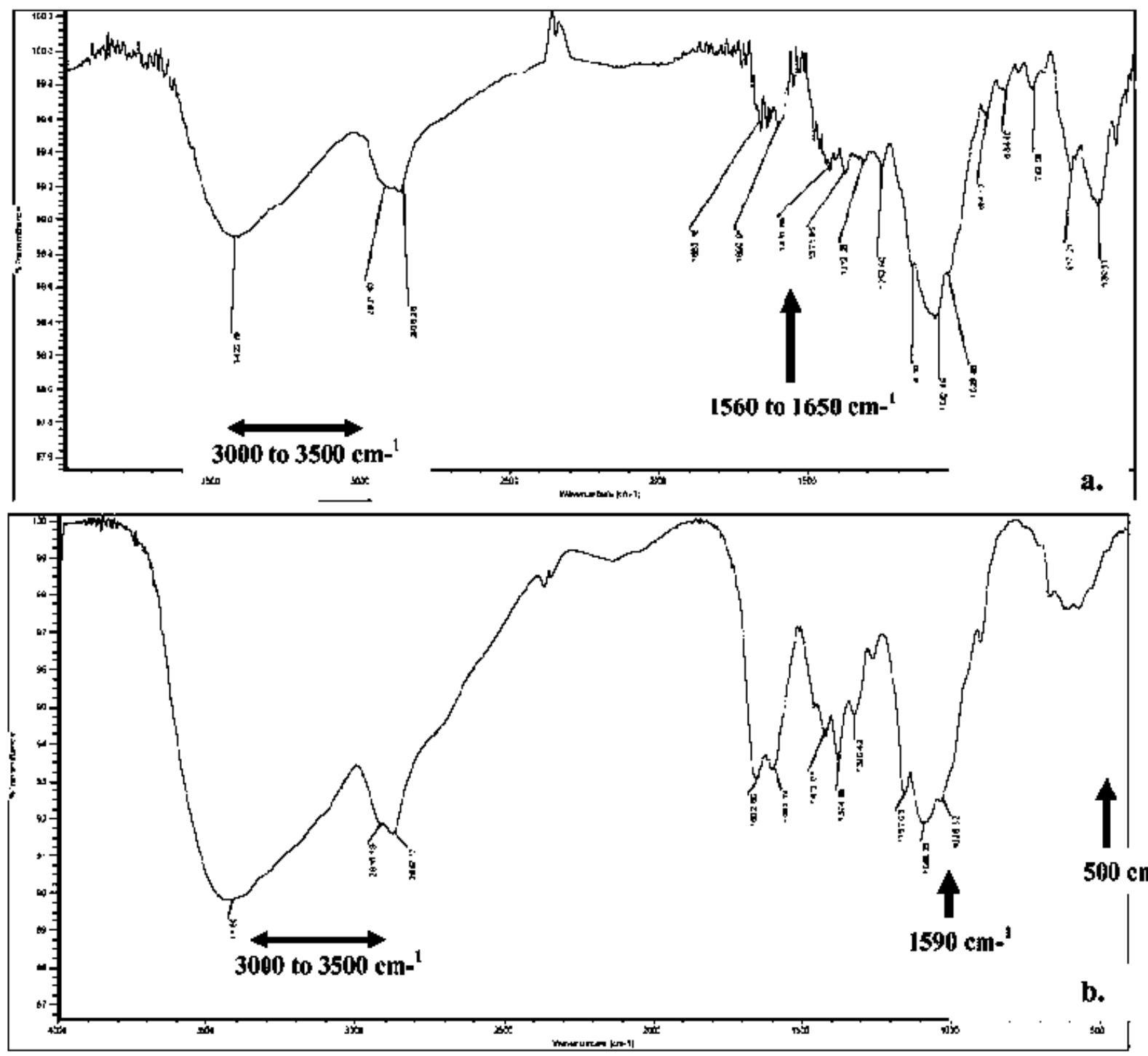

Figure 2. IR spectra of unmodified chitosan and crosslinked chitosan. Percent transmittance $(\% T)$ is denoted on the $Y$ axis and wavelengths (in $\mathrm{cm}^{-1}$ ) are denoted on the $X$ axis. Relevant wavelengths and regions are indicated with arrows. [Color figure can be viewed in the online issue, which is available at www.interscience.wiley.com.] 


\section{Mechanical properties (compression test)}

It is expected that crosslinked films and scaffolds will possess higher stiffness and elastic modulus. The results show that chemically crosslinked films had a 1.5-times higher modulus than uncrosslinked films. The elastic moduli of the crosslinked scaffolds can be seen in Figure 4. The crosslinking process significantly increased the elastic modulus of the chitosan substrate $(p<0.01)$, indicating an increase in stiffness after crosslinking. The crosslinked scaffolds $(0.5<$ to $5<)$ have about $2-5$ times higher elastic modulus $(7.4-19.9$ $\mathrm{kPa})$ compared to uncrosslinked scaffolds $(3.8 \mathrm{kPa})$. Chondrocytes, when seeded on flexible substrates (like collagen), have been shown to exert contractile forces.

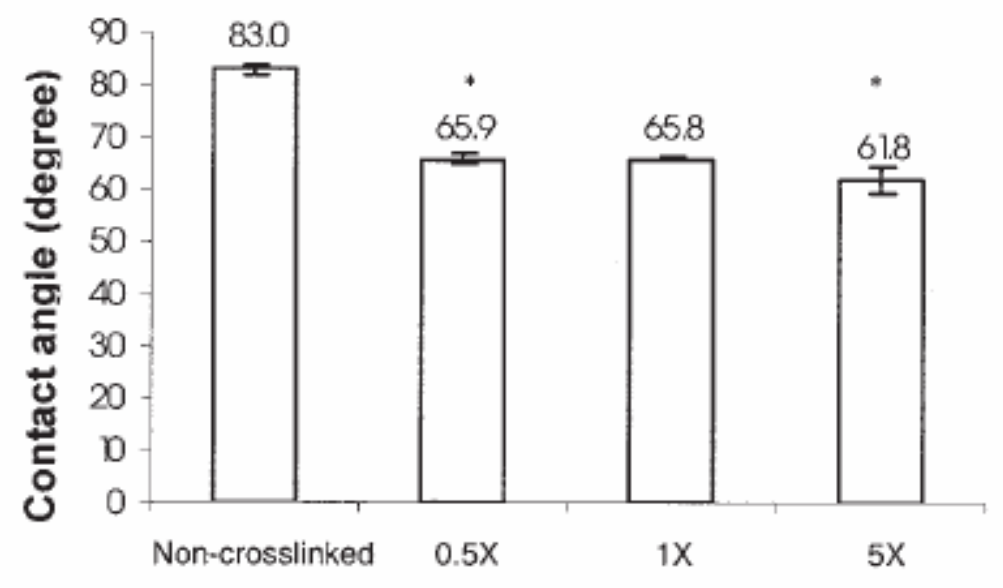

Molar ratio of ether to chitosan amino group

Figure 3. Contact angle of uncrosslinked and crosslinked chitosan films. Chitosan films were washed and an OCA-20 was used to measure the contact angles. A minimum of three drops were collected for each liquid used at different locations on the sample surface and mean error was reported. 


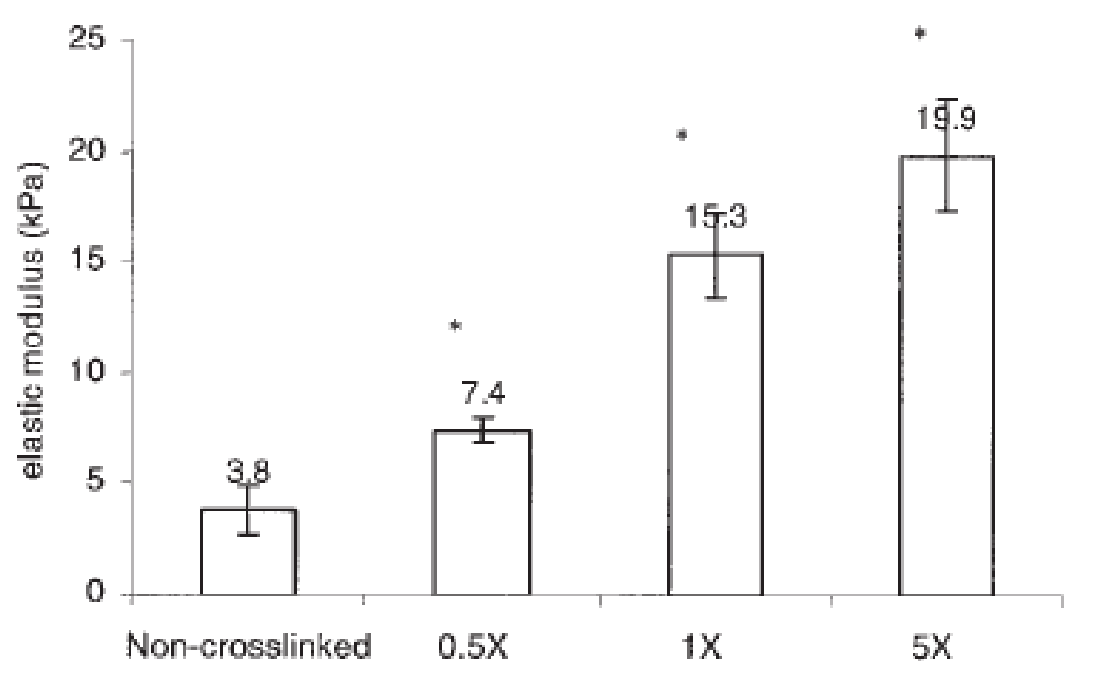

Molar ratio of ether to chitosan amino group

\section{Figure 4.}

Elastic modulus of scaffolds prepared by the FDL method in hydrated state. The test was done by an Instron using a 50-kgf load cell with crosshead moving at $2 \mathrm{~mm} / \mathrm{min}$.

Seeded scaffolds have undergone a $10-30 \%$ reduction in diameter.16,17 The diameter of unseeded substrates (no cells) based on chitosan changed less than $2 \%$ during the threeweek culture period. The present results (data not included) show that the spreading and adhesion of chondrocytes on films and scaffolds prepared by the FDL method did not deform the gels or shrink the surfaces. This may be explained by the difference in the Young's modulus of these materials. The substrates used in these studies had higher elastic modulus when compared to the flexible collagen substrates with reported Young's modulus of $100-150 \mathrm{~Pa}$. This result is supported by the observation that the morphology of the scaffold, as judged by SEM, and the porosity of the scaffolds did not undergo a significant change upon crosslinking (data not included). 


\section{Degradation with chitosanase}

The degradation results of uncrosslinked and crosslinked chitosan scaffolds are shown in Table I. The noncrosslinked scaffolds were completely digested in $24 \mathrm{~h}$. The initial $(<4 \mathrm{~h})$ degradation of the more crosslinked samples was slower than the less- or noncrosslinked ones. However, by the end of $24 \mathrm{~h}$, the amount of chitosan digested was not different between different degrees of crosslinked chitosan scaffolds. All chitosan samples were degraded over the 24-h period by chitosanase. More crossed chitosan had a slower initial $(<4 \mathrm{~h})$ degradation rate than the less crosslinked ones. Chitosanase-assisted hydrolysis requires that beta-1,4-linkages between $\mathrm{N}$-acetyl-dglucosamine and $\mathrm{d}$ glucosamine residues in chitosan be presented.41 Chemical modification of chitosan at the amino group may render the glucosamine residues inaccessible to chitosanase, or the crosslinked segments may form a conformation that hinders chitosanase from recognizing other active moieties. As no significant differences in the rate or extent of degradation were observed between uncrosslinked and crosslinked chitosan films, it is concluded that the method of crosslinking did not alter the enzymatic susceptibility. It is estimated that at the highest ratio of the functional diepoxide to the $\mathrm{ONH} 2$ groups on the chitosan molecule, only about $25-35 \%$ of the available amino groups were modified, and that at this level of modification the enzymatic susceptibility was not altered.

\section{Cytotoxicity of crosslinked chitosan surfaces}

The in vitro biocompatibility of crosslinked films and scaffolds prepared in the laboratory were evaluated to ascertain the impact, if any, of chemical crosslinkers used. The ability 
of crosslinked substrates to support growth and proliferation of chondrocytes is presented in Table II. The absorbance from the cell viability test (MTT assay) on various degrees of crosslinked films was calculated into the percentage of the control (cells on tissue-culture polystyrene, TCP). The viability of cells on the crosslinked chitosan films was not significantly different from the cells on the TCP. There was no significant difference in viability between different degrees of crosslinked films. The results show that the modified surface was not cytotoxic to the chon drocyte cells, because the cell viability remained the same as the control after 3 days in culture. Cell viability in the range of 90 $97 \%$ was routinely attained, indicating that modification is not cytotoxic and the crosslinked chitosan scaffold maintained biocompatibility.

\section{TABLE I \\ Degradation of Chitosan Scaffolds in Chitosanase Acetate Solution $(0.1 \mathrm{U} / \mathrm{mL})$}

\begin{tabular}{crccc}
\hline \multirow{2}{*}{$\begin{array}{c}\text { Sampling } \\
\text { Tinne }\end{array}$} & \multicolumn{4}{c}{ Coumples (\% Degradation) } \\
\cline { 2 - 5 } & \multicolumn{1}{c}{$0.5 \times$} & $1 \times$ & $5 \times$ \\
\hline 1 hour & $42.3 \pm 2.0$ & $45.6 \pm 8.7$ & $44.0 \pm 7.1$ & $27.9 \pm 5.6$ \\
2 hours & $72.7 \pm 4.7$ & $66.4 \pm 7.7$ & $54.6 \pm 0.6$ & $32.4 \pm 1.7$ \\
4 hours & $95.4 \pm 3.7$ & $81.5 \pm 0.9$ & $70.1 \pm 0.8$ & $59.8 \pm 0.2$ \\
24 hours & $100.0 \pm 0.0$ & $89.3 \pm 5.8$ & $95.3 \pm 3.2$ & $94.1 \pm 21$ \\
\hline
\end{tabular}

Unit is in percentage of weight loss based on initial dry weight. Numbers are presented in average \pm standard deviation (Ave $\pm S D$ ). 
TABLE II

Cell Viability on Crosslinked Chitosan Films

\begin{tabular}{cccccc}
\hline Surface & TCP & $0 \times$ & $0.5 \times$ & $1 \times$ & $5 \times$ \\
\hline Viability (\% of control) & $100.0 \pm 12.0$ & $95.4 \pm 2.7$ & $94.2 \pm 6.7$ & $90.9 \pm 12.4$ & $96.8 \pm 6.2$ \\
\hline Unit is in \% TCP control $\left[=\left(A_{595}\right.\right.$ of samples $/ A_{595}$ of TCP $\left.) \times 100 \%\right] .0 .5 \times, 1 \times$, and $5 \times$ are the molar ratio of diepoxide to amino \\
groups of the film. Numbers are presented in average \pm standard deviation (Ave \pm SD).
\end{tabular}

\section{Cell morphology and proliferation}

Mechanical forces can arise from two sources-the environment and contractile forces generated by the cells, wherein cells provide the initial input and the substrate reacts to it by deforming passively. It is hypothesized that chondrocytes can sense their intrinsic mechanical environment and that substrate or scaffold rigidity/stiffness can also serve as an intrinsic mechanical stimuli. It is expected that chondrocyte will possess different morphologies when seeded on flexible substrates as opposed to stiffened substrates. Crosslinked films may be able to direct the chondrocytes to possess morphology that resembles Type IV classification42 when compared to uncrosslinked surfaces. The SEM images of chondrocytes grown on free swelling films, after 1 week of culture, are shown in Figure 5. When seeded on noncrosslinked and crosslinked chitosan films and observed 1 week after seeding, the cells possessed a flattened and spread morphology. This result agrees with the previous finding, where seeded chondrocytes possessed Type IV morphology on planar surfaces. 26,38 At the end of a week of culture, cell proliferation appeared to be higher on crosslinked films $(9.2<104$ to $12.6<104$ cells per film) when compared to noncrosslinked films $(6.5<1.1<104$ cells per film). The SEM images of chondrocytes growing on FDL scaffolds are shown in Figure 6. 


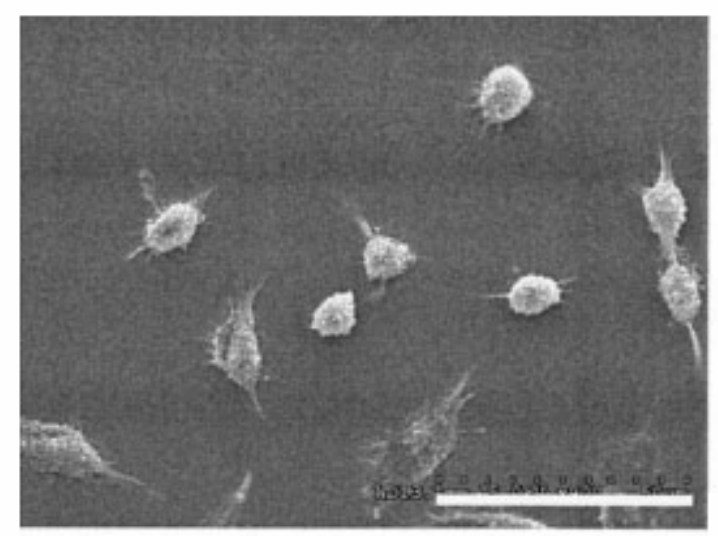

(A)

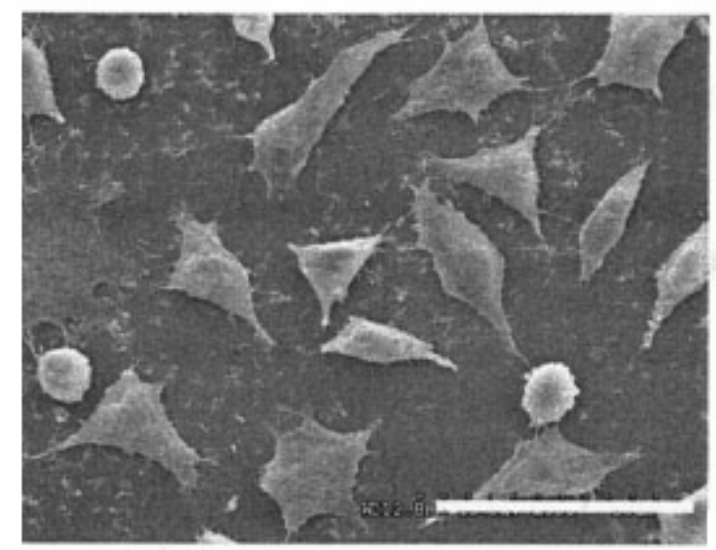

(C)

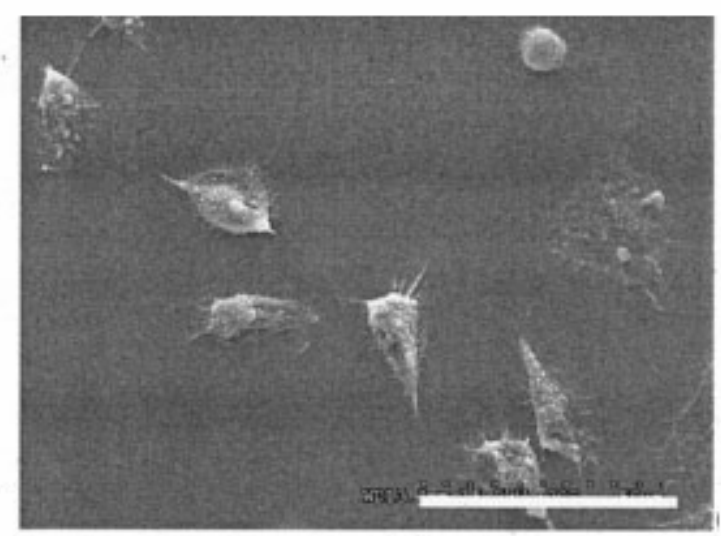

(B)

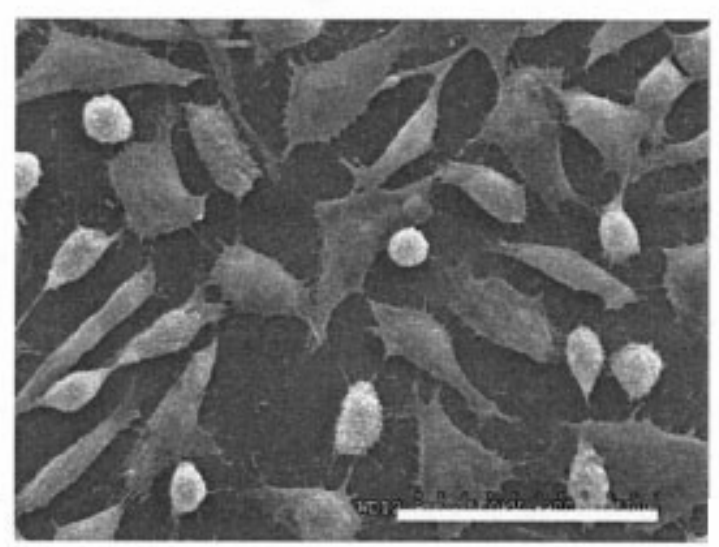

(D)

\section{Figure 5.}

SEM micrograph of chondrocytes seeded on uncrosslinked and crosslinked films. Representative SEM pictures of canine chondrocyte cells (passage 2) on (A) noncrosslinked (0<), (B) $0.5<,(C) 1<$, and (D) $5<$ crosslinked chitosan films. SEM micrographs were taken after 1 week of culture. The initial seeding density was $1<104$ cells/cm2. The scale bar is $50<m$. 


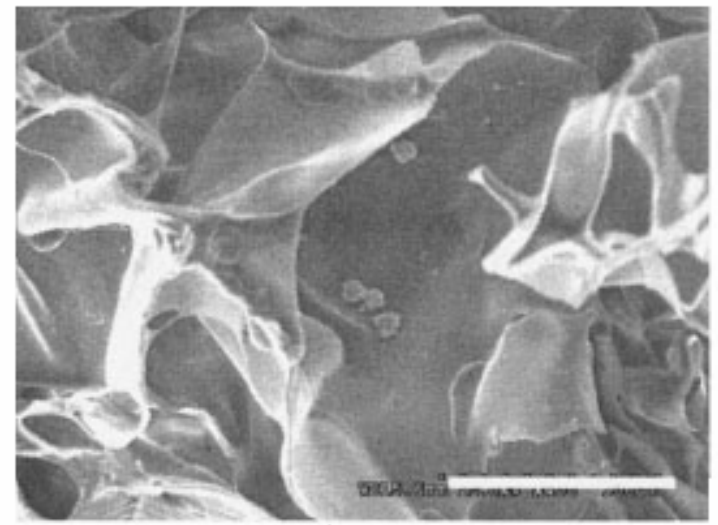

(A)

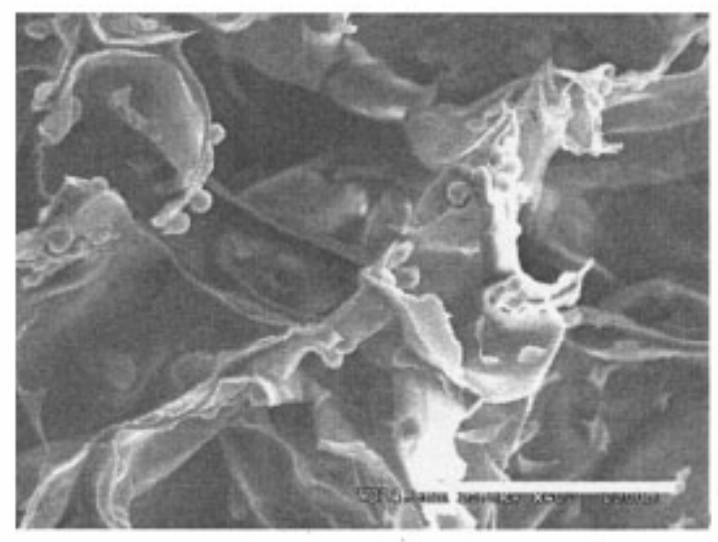

(C)

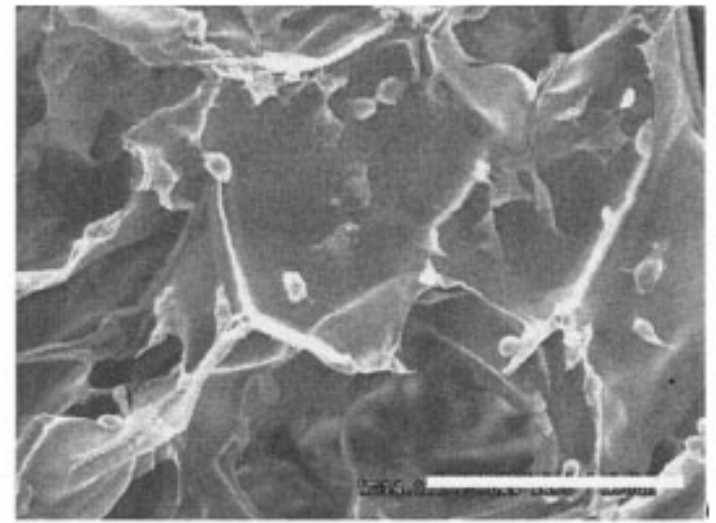

(B)

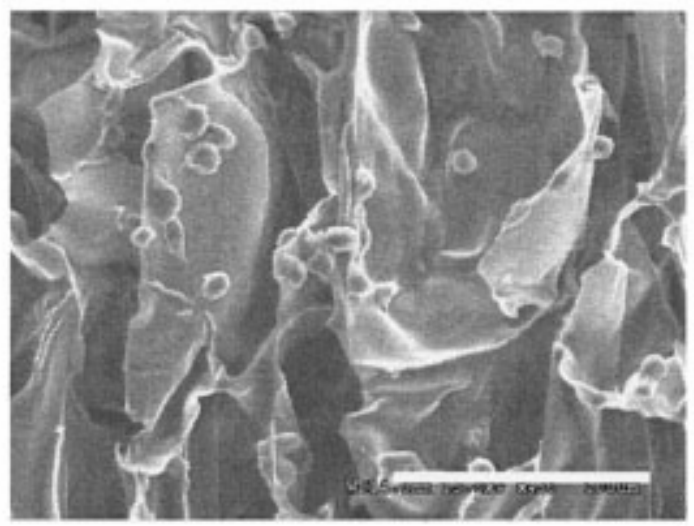

(D)

Figure 6. SEM micrograph of chondrocytes seeded on noncrosslinked and crosslinked scaffolds. Representative SEM pictures of canine chondrocyte cells (passage 3) on (A) noncrosslinked, (B) $0.5<,(C) 1<,(D) 5<$ crosslinked chitosan scaffolds are shown. SEM micrographs were taken after 1 week of culture. The scale bar is $100<m$. Cells appeared to have rounded chondrocytic morphology. 


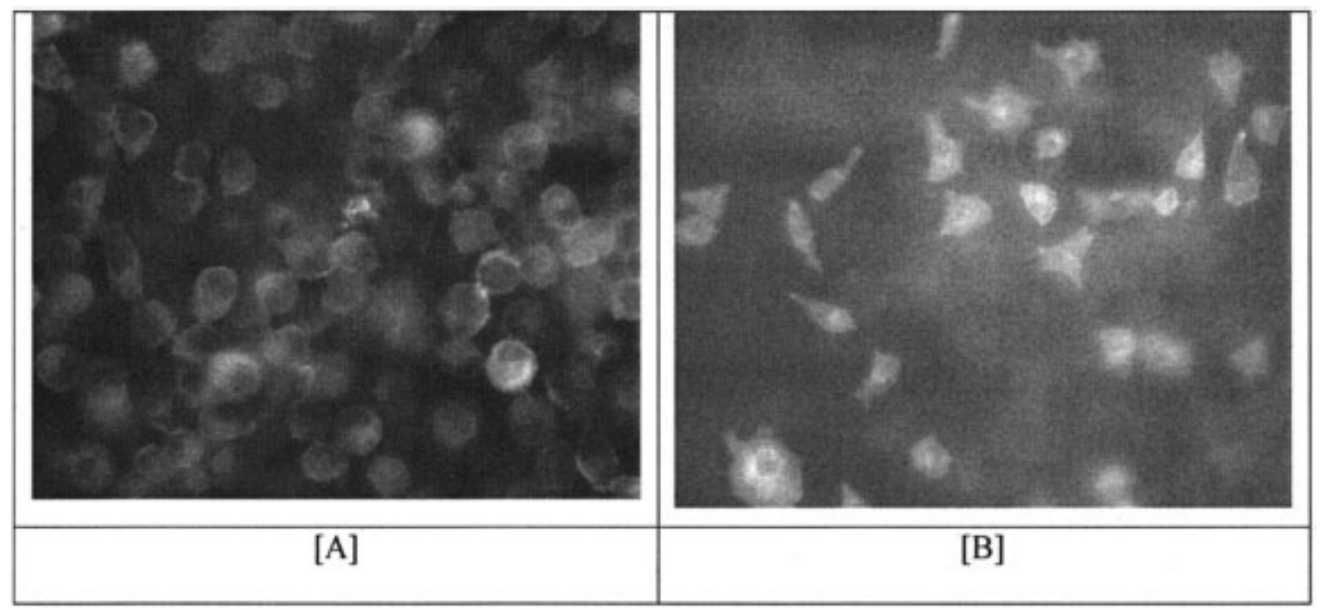

Figure 7. Morphologies of chondrocytes seeded on uncrosslinked and crosslinked chitosan films. Representative fluorescent pictures of canine chondrocyte cells (passage 3) of (A) on a noncrosslinked film and (B) on a $0.5<$ film. Cell and cytoskeletal morphology were assessed with the use of rhodamine-conjugated phalloidin to visualize filamentous actin. The initial seeding density was $1<104 \mathrm{cells} / \mathrm{cm} 2$, and cultures were maintained for 2 weeks. Actin filaments were detected by staining with TRITC conjugated phalloidin, and cells were observed by epifluorescence microscopy. The scale bar is $50<m$. Cells on uncrosslinked surfaces were nebulous, and cells on crosslinked surfaces appeared to have Type IV, flattened morphology.

Chondrocyte cells seeded on chitosan scaffolds proliferated, possessing a rounded chondrocytic morphology that is indicative of cells retaining their phenotypes. From the SEM pictures, it is concluded that at the end of a week of culture, crosslinked chitosan scaffolds [Fig. 6(B-D)] had a higher cell proliferation when compared to the noncrosslinked surfaces [Fig. 6(A)] as determined by the total amount of doublestranded 
DNA (dsDNA) detected in the digests. Uncrosslinked, $0.5<, 1<$, and $5<$ crosslinked scaffolds gave $57.0<7.7,68.7<0.8,103.2<4.6$, and $110.8<6.6 \mathrm{ng}$ of dsDNA $/ \mathrm{mL}$, respectively. Measurement of the cell count (data not included) showed a similar trend. The present observations suggest that chondrocytes growing on a 3D hydrogel scaffold have proper cell- cell contact with neighboring chondrocytes, whereas the chondrocytes growing on two dimensional films express flat and spread morphology. These results agree with previous studies, where chondrocytes appear to be rounded in three dimensional scaffolds while maintaining their phenotypes. 44-46 Previous studies have shown that it is necessary for chondrocytes to assume a spherical morphology in order to express the chondrocytic phenotype (Type II collagen and GAGs).45,46 This spherical morphology is also an indication of chondrocyte differentiation.46 In an extended experiment, chondrocytes, when maintained on non crosslinked scaffold for 21 days, gave $0.15<0.05<\mathrm{g}$ of $\mathrm{GAG} / \mathrm{mL}$ and chondrocytes maintained on $0.5<, 1<$, and $5<$ crosslinked scaffolds for a month gave $0.52<0.13,0.52<0.07$, and $0.69<0.04<\mathrm{g}$ of $\mathrm{GAG} / \mathrm{mL}$, respectively.

Chondrocytes were seeded onto crosslinked and uncrosslinked films and scaffolds, and the cytoskeletal morphologies were observed upon rhodamine- phalloidin staining of actin filaments of seeded chondrocytes. Representative morphologies obtained on noncrosslinked and crosslinked chitosan films membranes are shown in Figure 7. About $90 \%$ of the cells on uncrosslinked films had a spherical morphology and nebulous, punctate actin. Cells on the crosslinked films were flattened with stress fibers and $40-50 \%$ of cells surveyed had a Type-IV flattened morphology.42 Thus it appears that stiffer substrates directed the cells to possess a flattened morphology. Representative morphologies obtained on noncrosslinked and crosslinked chitosan scaffolds are shown in Figure 8. As observed by the fluorescent staining; a higher rate of proliferation was 
seen on crosslinked scaffolds [Fig. $8(B, C)$ ] when compared to noncrosslinked scaffolds [Fig. 8(A)].

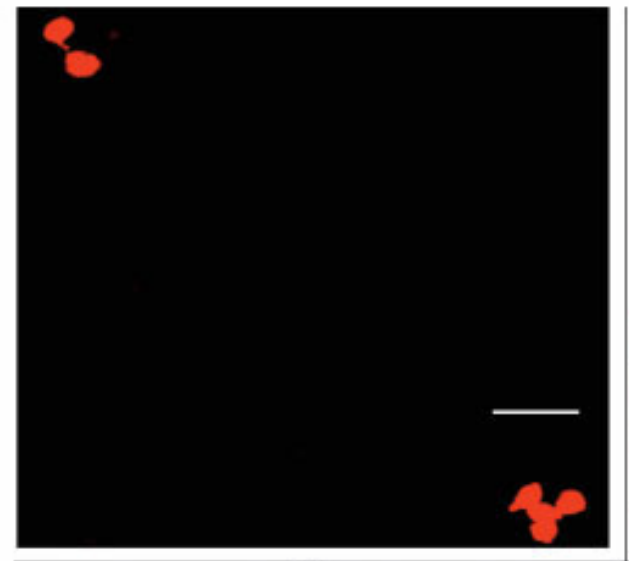

$[\mathrm{A}]$

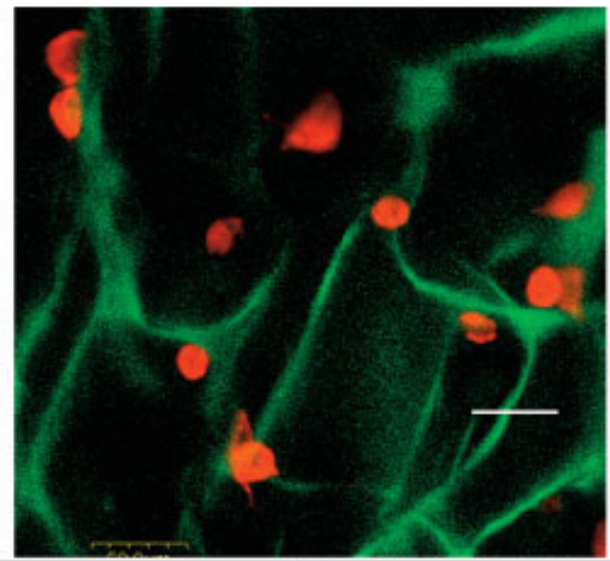

[B]

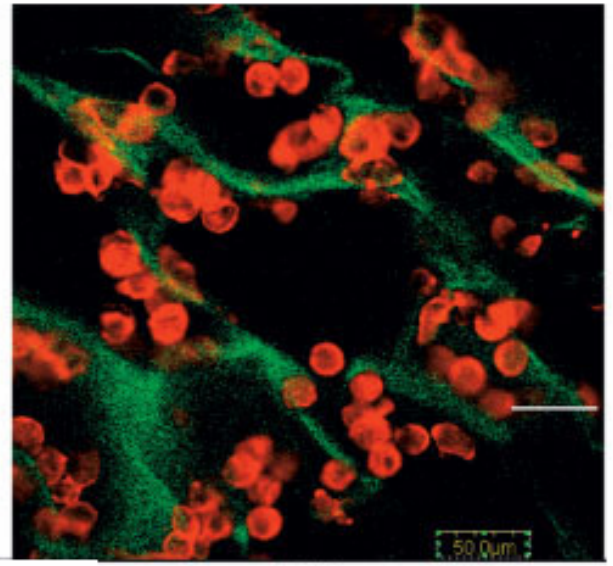

$[\mathrm{C}]$

Figure 8. A rhodamine-phalloidin stain of chondrocytes seeded on uncrosslinked and crosslinked scaffolds. Representative fluorescent pictures of canine chondrocyte cells (passage 3) (A) on a noncrosslinked scaffold; (B) on a $0.5<$ crosslinked scaffold, and (C) on a $5<$ crosslinked chitosan scaffolds. The initial seeding density was $3<105$ cells $/ \mathrm{mL}$ (2 $m L$ of cell suspension added per well) and cultures were maintained for 2 weeks. Actin filaments were detected by staining with TRITC conjugated phalloidin, and cells were observed by epifluorescence microscopy. The scale bar is $50<m$.

\section{CONCLUSION}

The images of cells from SEM and the fluorescent stain indicate that stiffer surfaces promote cell proliferation. The elastic moduli of crosslinked scaffolds were $100-400 \%$ more than that of the noncrosslinked scaffold. In the absence of any external stimuli, it is postulated that the rigidity of the films might be the factor enhancing chondrocyte cell growth 
and biosynthetic activity. In conclusion, it has been shown that the chemical crosslinking strategy used imparts additional matrix stiffness without impacting the biocompatibility or the cell adhesive properties of the films or scaffolds. The augmented mechanical strength may provide additional resistance to cell-mediated scaffold shrinkage and pore size change. Finally, the increase in substrate stiffness appears to serve as an intrinsic stimulus that promotes cell proliferation and preservation of morphology. The slower enzyme degradation rate of crosslinked scaffolds can be viewed as an advantage for a degradable material, because the degradation rate can thus be further modified to suit various tissue recovery time in vivo. This study provides an alternative way to control the physical properties of implant materials and to improve chondrocyte proliferation. Continuing studies aim to assess the phenotype and biosynthetic activity of chondrocytes on the surfaces evaluated in the present study.

\section{References}

1. LeBaron RG, Athanasiou KA. Ex vivo synthesis of articular cartilage. Biomaterials 2000;21:2575-2587.

2. Nehrer S, Breinan HA, Ramappa A, Hsu HP, Minas T, Shortkroff S, Sledge CB, Yannas IV, Spector M. Chondrocyte-seeded collagen matrices implanted in a chondral defect in a canine model. Biomaterials 1998;16:2313-2328.

3. Nehrer S, Breinan HA, Ramappa A, Shortkroff S, Young G, Minas T, Sledge CB, Yannas IV, Spector M. Canine chondrocytes seeded in type I and type II collagen implants investigated in vitro. J Biomed Mater Res 1997;38:95-104.

4. Hung CT, Mauck RL, Wang CC, Lima EG, Ateshian GA. A paradigm for functional tissue engineering of articular cartilage via applied physiologic deformational loading. Ann Biomed Eng 2004;32:35- 49 . 
5. Kimura T, Yasui N, Ohsawa S, Ono K. Chondrocytes embedded in collagen gels maintain cartilage phenotype during long term cultures. Clin Orthop 1984;186:231.

6. Aulthouse AL, Beck M, Griffey E, Sanford J, Arden K, Machado MA, Horton WA. Expression of the human chondrocyte phenotype in vitro. In Vitro Cell Dev Biol 1989;25:659-668.

7. Sechriest VF, Miao YJ, Niyibizi C, Westerhausen-Larson A, Matthew HW, Evans CH, Fu FH, Suh JK. GAG-augmented polysaccharide hydrogel: a novel biocompatible and biodegradable material to support chondrogenesis. J Biomed Mater Res 2000; 49:534 -541.

8. Klokkevold PR, Vandemark L, Kenney EB, Bernard GW. Osteogenesis enhanced by chitosan (poly-N-acetyl glucosaminoglycan) in vitro. J Periodontol 1996;67:1170 -1175.

9. Muzzarelli RA, Mattioli-Belmonte M, Tietz C, Biagini R, Ferioli G, Brunelli MA, Fini M, Giardino R, Ilari P, Biagini G. Stimulatory effect on bone formation exerted by a modified chitosan.

Biomaterials 1994;15:1075-1081.

10. Hirano S. Chitin biotechnology applications. Biotechnol Annu Rev 1996;2:237-258.

11. Muzzarelli MAA, Ilari P, Tomasetti MB. J Biomater Sci Polym Ed 1994;6:541.

12. Madihally SV, Matthew HWT. Porous chitosan scaffolds for use in tissue engineering. Biomaterials $1999 ; 20: 1133$.

13. Shanmugasundaram N, Ravichandran P, Neelakanta Reddy P, Ramamurty N, Pal S, Panduranga Rao K. Biomaterials 2001;22: 1943-1951.

14. VandeVord PJ, Matthew HWT, DeSilva SP, Wu B, Wooley PH. Evaluation of the biocompatibility of a chitosan scaffold in mice. J Biomed Mater Res 2002;59:585-590.

15. Lee JE, Kim SE, Kwon IC, Ahn HJ, Cho H, Lee SH, Kim HJ, Seong SC, Lee MC. Effects of a chitosan scaffold containing TGF-beta1 encapsulated chitosan microspheres on in vitro chondrocyte culture. Artif Organs 2004;28:829-839.

16. Toolan BC, Frenkel SR, Pachence JM, Yalowitz L, Alexander H. Effects of growth-factorenhanced culture on a chondrocyte- collagen implant for cartilage repair. J Biomed Mater Res 1996; 31:273-280. 
17. Hsu SH, Whu SW, Hsieh SC, Tsai CL, Chen DC, Tan TS. Evaluation of chitosan-alginatehyaluronate complexes modified by an RGD-containing protein as tissue-engineering scaffolds for cartilage regeneration. Artif Organs 2004;28:693- 703.

18. Mauck RL, Seyhan SL, Ateshian GA, Hung CT. Influence of seeding density and dynamic deformational loading on the developing structure/function relationships of chondrocyteseeded agarose hydrogels. Ann Biomed Eng 2002;30:1046 - 1056.

19. Chowdhury TT, Salter DM, Bader DL, Lee DL. Integrin-mediated mechanotransduction processes in TGF<-stimulated monolayer expanded chondrocytes. Biochem Biophys Res Commun 2004;318:873- 881.

20. Shelton JC, Bader DL, Lee DA. Mechanical conditioning influences the metabolic response of cell-seeded constructs. Cells Tissues Organs 2003;175:140 -150.

21. Lee CR, Grodzinsky AJ, Spector M. Biosynthetic response of passaged chondrocytes in a type II collagen scaffold to mechanical compression. J Biomed Mater Res A 2003;64:560 - 569.

22. Lee CR, Grodzinsky AJ, Spector M. The effects of crosslinking of collagen-glycosaminoglycan scaffolds, on compressive stiffness, chondrocyte-mediated contraction, proliferation and biosynthesis. Biomaterials 2001;22:3145-3154.

23. Zaleskas JM, Kinner B, Freyman TM, Yannas IV, Gibson LJ, Spector M. Contractile forces generated by articular chondrocytes in collagen-glycosaminoglycan matrices. Biomaterials 2004;25:1299-1308.

24. Nehrer S, Breinan HA, Ramappa A, Young G, Shortkroff S, Louie LK, Sledge CB, Yannas IV, Spector M. Matrix collagen type and pore size influence behaviour of seeded canine chondrocytes. Biomaterials 1997;18:769 -776.

25. Sellers RS, Peluso D, Morris EA. The effect of recombinant human bone morphogenetic protein-2 (rhBMP-2) on the healing of full-thickness defects of articular cartilage. J Bone Joint Surg Am 1997;79:1452-1463. 
26. Sellers RS, Zhang R, Glasson SS, Kim HD, Peluso D, D’Augusta DA, Beckwith K, Morris EA. Repair of articular cartilage defects one year after treatment with recombinant human bone morphogenetic protein-2 (rhBMP-2). J Bone Joint Surg Am 2000;82: 151-160.

27. Hunter CJ, Mouw JK, Levenston ME. Dynamic compression of chondrocyte-seeded fibrin gels: effects on matrix accumulation and mechanical stiffness. Osteoarthritis Cartilage $2004 ; 12: 117-130$.

28. Wang Q, Breinan HA, Hsu HP, Spector M. Healing of defects in canine articular cartilage: distribution of nonvascular alphasmooth muscle actin-containing cells. Wound Repair Regen 2000;8:145-158.

29. Ma L, Gao C, Mao Z, Zhou J, Shen J. Biodegradability and cell-mediated contraction of porous collagen scaffolds: the effect of lysine as a novel crosslinking bridge. J Biomed Mater Res A $2004 ; 71: 334-342$.

30. Torres DS, Freman TM, Yannas IV, Spector M. Tendon cell contraction of collagen-GAG matrices in vitro: effect of crosslinking. Biomaterials 2000;21:1607-1619.

31. Genes NG, Rowley JA, Mooney DJ, Bonassar LJ. Effects of substrate mechanics on chondrocyte adhesion to modified alginate surfaces. Arch Biochem Biophys 2004;422:161-167.

32. Roy SK, Todd JG, Glasser WG. Crosslinked hydrogel beads from chitosan. U. S. Patent No. 5770712; June 23, 1998.

33. Subramanian A, Vu D, Larsen GF, Lin HY. Preparation and evaluation of electrospun chitosan/PEO fibers for potential application in cartilage tissue engineering. J Biomater Sci Polym Ed 2005;16:861- 873.

34. Benjamin M, Hillen B. Mechanical influences on cells, tissues and organs-mechanical morphogenesis. Eur J Morphol 2003; 41:3-7

35. Yokota H, Goldring MB, Sun HB. CITED2-mediated regulation of MMP-1 and MMP-13 in human chondrocytes under flow shear. J Biol Chem 2003;278:47275-47280. 36. Wong M, Siegrist M, Goodwin K. Cyclic tensile strain and cyclic hydrostatic pressure differentially regulate expression of hypertrophic markers in primary chondrocytes. Bone 2003;33:685- 693. 
37. Rowin ME, Whatley RE, Yednock T, Bohnsack J F. Intracellular calcium requirements for beta1 integrin activation. J Cell Physiol 1998;175:193-202.

38. Akiyama SK. Integrins in cell adhesion and signaling. Hum Cell 1996;9:181-186.

39. Arcangeli A, Becchetti A, Mannini A, Mugnai G, De Filippi P, Tarone G, Del Bene MR, Barletta E, Wanke E, Olivotto M. Integrin-mediated neurite outgrowth in neuroblastoma cells depends on the activation of potassium channels. J Cell Biol 1993; 122:1131-1143.

40. Hornby JE. Measurements of cell adhesion. II. Quantitative study of the effect of divalent ions on cell adhesion. J Embryol Exper Morphol 1973;30:511-518.

41. Ohtakara A, Izume M, Mitsutomi M. Action of microbial chitinases on chitosan with different degrees of deacetylation. Agric Biol Chem 1988;52:3181-3182.

42. Massia SP, Hubbell JA. An RDG spacing of $440 \mathrm{~nm}$ is sufficient for integrin mediated fibroblast spreading and $140 \mathrm{~nm}$ for focal contact and stress fiber formation. J Cell Biol 1991;114:1089-1100.

43. Elsdale T, Bard J. Collagen substrata for studies on cell behavior. J Cell Biol 1972;54:626637.

44. Benya PD, Shaffer JD. Dedifferentiated chondrocytes reexpress the differentiated collagen phenotype when cultured in agarose gels. Cell 1982;30:215-224.

45. Risbud M, Ringe J, Bhonde R, Sittinger M. In vitro expression of cartilage-specific markers by chondrocytes on a biocompatible hydrogel: implications for engineering cartilage tissue. Cell Transplant 2001;10:755-763.

46. Veilleux NH, Yannas IV, Spector M. Effect of passage number and collagen type on the proliferative, biosynthetic, and contractile activity of adult canine articular chondrocytes in type I and II collagen-glycosaminoglycan matrices in vitro. Tissue Eng 2004;10:119 -127. 